\title{
Contracts for Modular Discrete Controller Synthesis *
}

\author{
Gwenaël Delaval \\ INRIA, Grenoble, France \\ gwenael.delaval@inria.fr
}

\author{
Hervé Marchand \\ INRIA, Rennes, France \\ herve.marchand@inria.fr
}

\author{
Eric Rutten \\ INRIA, Grenoble, France \\ eric.rutten@inria.fr
}

\begin{abstract}
We describe the extension of a reactive programming language with a behavioral contract construct. It is dedicated to the programming of reactive control of applications in embedded systems, and involves principles of the supervisory control of discrete event systems. Our contribution is in a language approach where modular discrete controller synthesis (DCS) is integrated, and it is concretized in the encapsulation of DCS into a compilation process. From transition system specifications of possible behaviors, DCS automatically produces controllers that make the controlled system satisfy the property given as objective. Our language features and compiling technique provide correctness-by-construction in that sense, and enhance reliability and verifiability. Our application domain is adaptive and reconfigurable systems: closed-loop adaptation mechanisms enable flexible execution of functionalities w.r.t. changing resource and environment conditions. Our language can serve programming such adaption controllers. This paper particularly describes the compilation of the language. We present a method for the modular application of discrete controller synthesis on synchronous programs, and its integration in the BZR language. We consider structured programs, as a composition of nodes, and first apply DCS on particular nodes of the program, in order to reduce the complexity of the controller computation; then, we allow the abstraction of parts of the program for this computation; and finally, we show how to recompose the different controllers computed from different abstractions for their correct co-execution with the initial program. Our work is illustrated with examples, and we present quantitative results about its implementation.
\end{abstract}

Categories and Subject Descriptors D.3.3 [Programming Languages]: Language Constructs and Features-Control structures; C.3 [Special-purpose and Application-based Systems]: Real-time and embedded systems; D.2.2 [Software Engineering]: Design Tools and Techniques-Computer-aided software engineering, State diagrams; D.2.4 [Software Engineering]: Software / Program Verification-Formal methods, Programming by contract

\section{General Terms Design, Languages, Reliability, Verification}

Keywords Discrete controller synthesis, modularity, components, contracts, reactive systems, synchronous programming, adaptive and reconfigurable systems

\footnotetext{
* This work was partially supported by the Minalogic project MIND.
}

Permission to make digital or hard copies of all or part of this work for personal or classroom use is granted without fee provided that copies are not made or distributed for profit or commercial advantage and that copies bear this notice and the full citation on the first page. To copy otherwise, to republish, to post on servers or to redistribute to lists, requires prior specific permission and/or a fee.

LCTES'10, April 13-15, 2010, Stockholm, Sweden.

Copyright (C) 2010 ACM 978-1-60558-953-4/10/04 .. \$10.00

\section{Motivations}

We integrate discrete controller synthesis (DCS) into a modular compilation process for a synchronous language: BZR, with motivations concerning the design of programming languages, the use of DCS, and the control of adaptive and reconfigurable systems.

Programming languages. We propose a compilation concretely exploiting a representation of the dynamical behavior of the program. Classically, compilation considers properties holding for all states (i.e., static); but we propose to consider state and path-dependent aspects (i.e., dynamical) [21].

DCS is a constructive operation, as it computes not a diagnostic about correctness, but a correct solution. A few works exist about its integration into a programming language framework [4]. We associate it to a contract mechanism, making it easier to use by programmers and favoring scalability (see further). Symmetrically, we propose a new point of view on the design by contracts principle: our programming language allows contracts to be enforced in nondeterministic programs, instead of being checked or proved correct.

Discrete controller synthesis. The modular application of DCS, which we are addressing in this paper, is based on contract enforcement and abstraction of components, with the aim of improving the scalability of the techniques devoted to DCS. Furthermore, the integration of these techniques in a high-level programming language also contributes to make it more widely usable in computer systems, and to study implementations of the controllers at a higher level than programmable logic controllers used in automation.

Control of adaptive and reconfigurable systems. Embedded systems have to be predictable, for safety-criticality issues. They also have to be able of dynamical adaptivity and reconfiguration, in reaction to environment changes, related to resources or dependability. This requires abilities for sensing the state of a system, deciding, based on a representation of the system, upon reconfiguration actions, and performing and executing them. These functionalities are assembled into a decision loop as illustrated in Figure 1(a).

Approach followed in our work. We want to combine these two different requirements, i.e., to be adaptive and predictable. Our

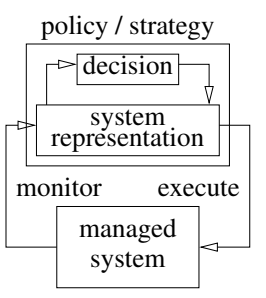

(a) Adaptive system.

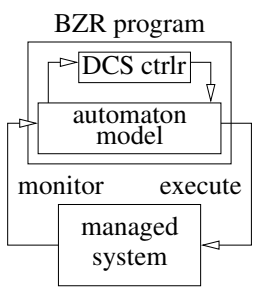

(b) BZR programming.
Figure 1. BZR programming of adaptation control. 


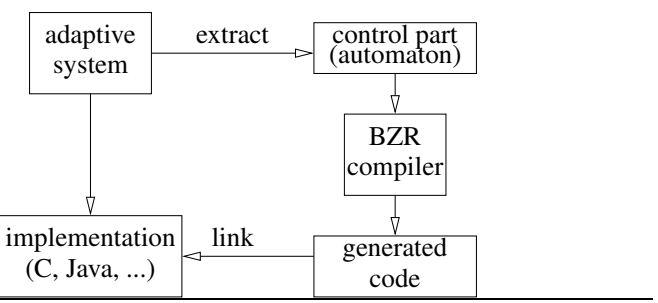

Figure 2. Development process using BZR.

programming language is specially suited for user-friendly design of safe control loops of adaptive systems, relying on discrete control theory techniques. It separates concerns, as illustrated in Figure 1(b), by supporting separate specification of, on one hand, the possible behaviors of the components, and on the other hand, the control objectives for the components assembly. From these two specifications, DCS can automatically generate, if a solution exists, a correct control decision component.

Contribution of the paper. Our precise contribution is the definition of a new construct, added to the HEPTAGON language, for defining behavioural contracts to be enforced on a node. The semantics of this new construct is defined in terms of a DCS problem, where resulting behaviours of the node are controlled in order to enforce the contract. We present the implementation of this language extension, involving a DCS tool encapsulated in the compilation.

The position of our contribution in the development process of an adaptive or reconfigurable system is illustrated in Figure 2. Our language is used for specifying the discrete control part of the system with automata. If explicitly defined in the host language, this part can be automatically extracted from the global specification by compilation; then the programmer does not need to know technicities of BZR or of DCS. Other, more data-related parts of the adaptive system are best developed in appropriate host languages. It is compiled with an encapsulated phase of DCS, relying upon its formal semantics. Executable code is generated towards target languages, e.g., C or Java. This code is then linked back into the concrete implementation of the system, with appropriate interfacing of the monitoring and adaptation execution functionalities provided by the platform. This way, the discrete feedback control loop of the computing system is in place as in Figure 1(b). We are currently working on instantiations of this development process at different levels, ranging from architecture-level reconfigurable FPGAs, through operating systems administration loops, through component-based middleware, to application-level aspects.

Outline of the paper. Section 2 briefly recalls notions upon which we base our contribution (synchronous mode automata, DCS). Section 3 describes the BZR programming language, extending the previous ones with a notion of contract. Section 4 then formally describes the compilation of this language, where DCS operations are applied upon nodes with contracts. Section 5 outlines implementation and Section 6 discusses performances and scalability.

\section{Context}

\subsection{Synchronous mode automata}

We place our work in the framework of reactive systems and synchronous programming [7], and adopt its classical basic notions. More particularly, we will adopt notations inspired from the Lucid Synchrone language, mixing dataflow and automata [10].

\subsubsection{Behavior}

For our examples, we consider programs expressed as synchronous Moore machines, with parallel and hierarchical composition.

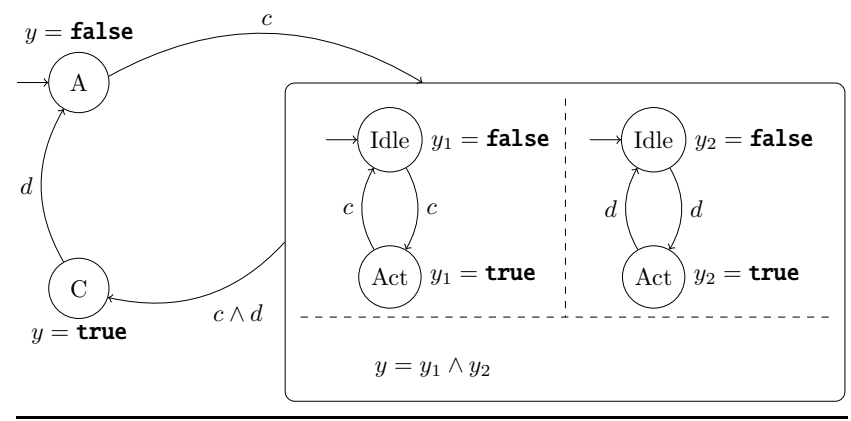

Figure 3. Mixed state / dataflow example

The states of such machines define data-flow equations, as in Lucid Synchrone or Lustre. At each step, according to inputs and current state values, equations associated to the current state produce outputs, and conditions on transitions are evaluated in order to determine the state for next step. Figure 3 gives an example. At the highest level, a three-state automaton is initially in state $A$, associated with equation $y=$ false. Upon condition $c$, it takes the transition to state $B$, itself associated with the parallel composition of three sub-nodes: two sub-automata and one simple equation, defining $y$ in terms of $y_{1}$ and $y_{2}$ defined in each of the sub-automata, by equations associated with the states, following the same principle as previously for state $A$. Upon $c \wedge d$, a transition is taken to state $C$, from where, upon $d$, another is taken to $A$.

\subsubsection{Structure}

For scalability and abstraction purpose, synchronous programs are structured in nodes, with a name $f$, inputs $x_{1}, \ldots, x_{n}$, outputs $y_{1}, \ldots, y_{p}$ and declarations $D . y_{i}$ variables are to be defined in $D$, using operations between values of $x_{j}$ and $y_{j}$. Figure 4 gives the graphical syntax of node definitions. The nodes are the abstraction level we will use in BZR to perform modular application of DCS.

The program of Figure 3 can then be structured as in Figure 5. The high-level automaton is specified in node defining $f$, with inputs $c$ and $d$, and output $y$, and with state $B$ is associated a an equation calling $g$. The latter is defined as a node with a body with three equations in parallel, two of which calling the node $h$. Finally, node $f$ is defined with a body containing a two-state automaton, with associated equations in the states.

\begin{tabular}{|c|}
\hline$f\left(x_{1}, \ldots, x_{n}\right)=y_{1}, \ldots, y_{p}$ \\
\hline$D$ \\
\hline
\end{tabular}

Figure 4. Node definition graphical syntax

\subsubsection{Corresponding transition system}

Behavior of such programs can be represented by a transition system, as illustrated in Figure 6, in its equational form. Synchronous compilers essentially compute this transition system from source programs, particularly handling the synchronous parallel composition of nodes. For a node $f$ as in Figure 4, a transition function Trans takes as inputs $X$ as well as the current state value, and produces the next state value. The latter is memorized by State for the next step. The output function Out takes the same inputs as $T$, and produces the outputs $Y$. We will use this representation to explain the notion of DCS, and to illustrate the behavior of our language.

\subsection{Discrete Controller Synthesis}

Discrete controller synthesis (DCS), emerged in the 80's [8, 20] allows to use constructive methods, that ensure, off-line, required 


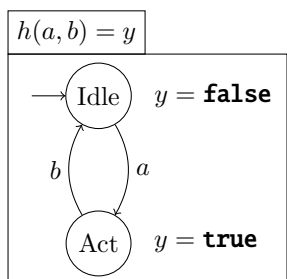

$$
\begin{gathered}
g(c, d)=y \\
\hline y_{1}=h(c, c) \\
---\bar{d},-\bar{d}) \\
y_{2}=h\left(d,-\bar{\wedge}-\overline{y_{2}}\right. \\
-\bar{y}=\overline{y_{1}}-\overline{y_{1}}
\end{gathered}
$$

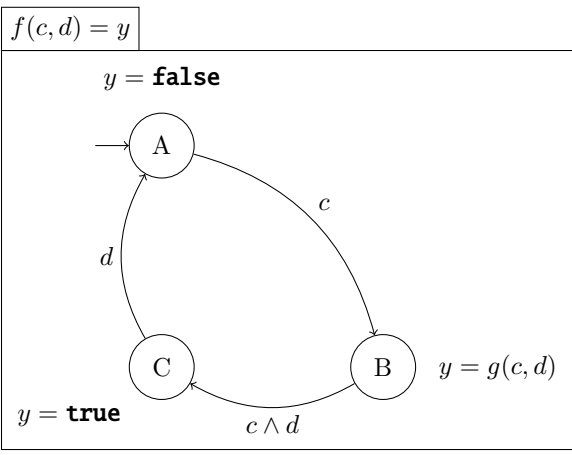

Figure 5. Structured program example

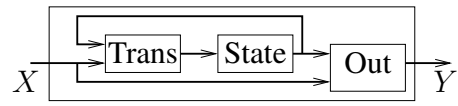

Figure 6. Transition system for a program

properties on the system behavior. DCS is an operation that applies on a transition system (originally uncontrolled), where inputs $X$ are partitioned into uncontrollable $\left(X^{u}\right)$ and controllable variables $\left(X^{c}\right)$. It is applied with a given control objective: a property that has to be enforced by control. In this work, we consider essentially invariance of a subset of the state space.

The purpose of DCS is to obtain a controller, which is a constraint on values of controllable variables $X^{c}$, function of the current state and the values of uncontrollable inputs $X^{u}$, such that all remaining behaviors satisfy the property given as objective. The synthesized controller is maximally permissive, it is therefore $a$ priori a relation; it can be transformed into a control function. This is illustrated in Figure 7, where the transition system of Figure 6, as yet uncontrolled, is composed with the synthesized controller $C t r l r$, which is fed with uncontrollable inputs $X^{u}$ and the current state value from State, in order to produce the values of controllables $X_{c}$ which are enforcing the control objective. The transition system then takes $X=X^{u} \cup X^{c}$ as input and performs a step.

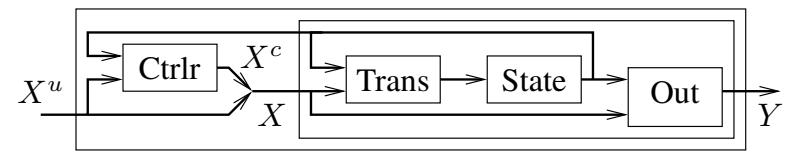

Figure 7. Controlled transition system

Modular DCS. In our approach, transitions systems are the starting point to model fragments of a large scale system, which usually consists of several composed and nested subsystems. To avoid state space explosion induced by the concurrent nature of the systems, there has been a growing interest in designing algorithms that perform the controller synthesis phase by taking advantage of the structure of the system without expanding the system. For the compositional aspect one can consider the works of [3, 11, 22]. More re- lated to our framework are the methods of $[1,15,14,18]$. However, their models is different from ours (asynchronous vs synchronous) as well as the way the low-level controlled system is abstracted in order to compute the controller of a higher level.

\subsection{Their combinations}

Previous work on the integration of DCS in a synchronous programming environment existed related to the Polychrony environment: SIGALI [17] is a tool that offers functionalities for verification of synchronous reactive systems and discrete controller synthesis. It manipulates Symbolic Transitions Systems (See Section 4.1), an equational and symbolic representation of automata. A wide variety of properties, such as invariance, reachability and attractivity, optimality w.r.t. to some quantitative criteria can be ensured by control. In the Polychrony environment, DCS is available as a formal tool amongst others, but not integrated at the programming language-level. A methodology for property-enforcing layers was proposed, [4] related to Mode Automata, but did not propose the language-level integration of objectives and DCS operations, as we do here. A deeper integration was proposed in a domain-specific language called NEMO [12], but it remained at the front-end of synchronous compilation, whereas this paper proposes full integration.

\subsection{Contracts, validation and DCS}

The notion of "design by contracts" have been introduced first in the Eiffel language [19]; contracts are require/ensure pairs on Eiffel functions which are then used at compilation time to add defensive code to these functions. The same design principle have been extended for reactive systems in [16], where reactive programs are given logical-time contracts, validated automatically by model-checking. We use here the same principle of logical-time contract, the difference with this latter work is essentially that our contracts are enforced by controller synthesis, instead of being validated. A more generic model of contracts has been proposed in [6], defining an algebra of contracts, which allows to consider the relation between sets of contracts defining one system, whereas our language only allows one contract to be associated to one node.

On the use of contracts for controller synthesis, [5] proposes a synthesis method based on the game theory, where contracts are used as assumptions to help the synthesis process. The modular aspect of the "design by contract" approach is not exploited.

Another related approach is interface synthesis [9]: the difference is that it is about constraining the environment of a component so that the component is used correctly, whereas in this work we constrain the component so that it works correctly whatever the environment does (within the assumptions) : the latter way is a more usual application of DCS. In particular, at the uppermost level, the assumption is taken as a hypothesis, to be checked by the programmer, in a way similar to the synchrony hypothesis, which has to be checked by the programmer on his system; on this basis the controller enforces the "guarantee" part. However, one can think of enforcing a guaranty condition at the top level but without any assumptions on the environment variables.

\section{The BZR language}

This section describes the original language construct which we add to the mode automata language introduced previously, and illustrates how to use it to design controllers in a modular way.

\subsection{Nodes with contracts}

We define basic contract nodes, and then composite contract nodes, and give the corresponding DCS problem solved at compilation. 


\subsubsection{Basic BZR node, with a contract}

Definition As illustrated in Figure 8, we associate to each node $f$ a contract, which is a program associated with two outputs : an output $e_{A}$ representing the environment model of the node and an invariance predicate $e_{G}$ that should be satisfied by the node. Its inputs are the inputs $x_{i}$ and outputs $y_{i}$ of the node $f$. At the node level, we assume the existence of a set $C=\left\{c_{1}, \ldots, c_{n}\right\}$ of controllable variables, that will be further used for ensuring this objective. This contract means that the node will be controlled, i.e., that values will be given to $c_{1}, \ldots, c_{n}$ such that, given any input trace yielding $e_{A}$ at each instant, the output trace will yield the true value for $e_{G}$ at each instant. This will be done by DCS. One can remark that the contract can itself feature a program, e.g., automata, observing traces, and defining states (for example an error state where $e_{G}$ is false, to be kept outside an invariant subspace). Also, one can define several such nodes with instances of the same body, that differ in assumptions and enforcements.

\begin{tabular}{|c|}
\hline$f\left(x_{1}, \ldots, x_{n}\right)=\left(y_{1}, \ldots, y_{p}\right)$ \\
\hline$e_{A} \Longrightarrow e_{G}$ \\
\hline with $c_{1}, \ldots, c_{q}$ \\
$y_{1}=f_{1}\left(x_{1}, \ldots, x_{n}, c_{1}, \ldots, c_{q}\right)$ \\
$\ldots$ \\
$y_{p}=f_{p}\left(x_{1}, \ldots, x_{n}, c_{1}, \ldots, c_{q}\right)$ \\
\hline
\end{tabular}

Figure 8. BZR node graphical syntax

Corresponding DCS problem For the compilation of such a BZR node, we will encode it into a DCS problem where, assuming $e_{A}$ (produced by the contract program, which will be part of the transition system), we will obtain a controller for the objective of enforcing $e_{G}$ (i.e., making invariant the sub-set of states where $e_{A} \Rightarrow e_{G}$ is true), with controllable variables $\mathrm{C}$.

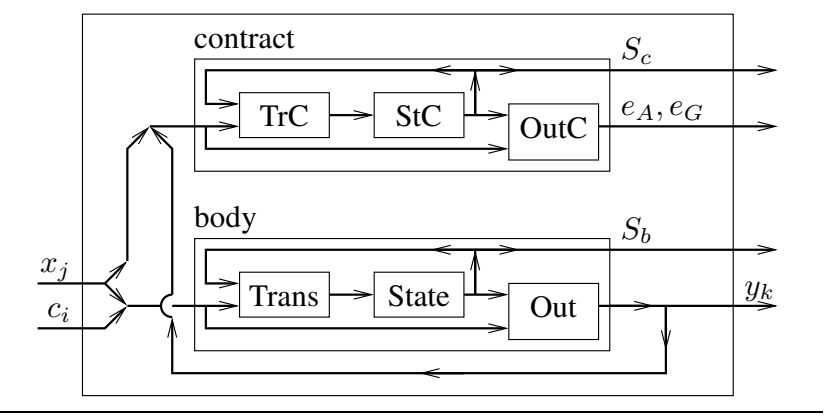

Figure 9. BZR node transition system

This is illustrated in Figure 9, re-using instances of the transition system of Figure 6: one for the contract (with transition function $\operatorname{TrC}$, state memory $S t C$ and output function $O u t C$ ) and one for the body of the node. The contract program has access to the node inputs $x_{1}, \ldots, x_{n}$ and outputs $y_{1}, \ldots, y_{p}$ of the body. Its outputs are $e_{A}$ and $e_{G}$, and the variables $c_{1}, \ldots, c_{q}$ are inputs of the body. We show explicitly the states $S_{c}$ and $S_{b}$ for clarity.

Figure 10 shows how the controller is synthesized and integrated to obtain the contract-enforcing node, similarly to Figure 7. The global state comprises both body and contract program state, and is taken as input by the controller, as well as the (uncontrollable) inputs $x_{1}, \ldots, x_{n}$ and contract Boolean outputs $e_{A}$ and $e_{G}$.

\subsubsection{BZR composite node}

Definition A composite BZR node has a contract of itself, and sub-nodes which are also BZR nodes, with their own contracts,

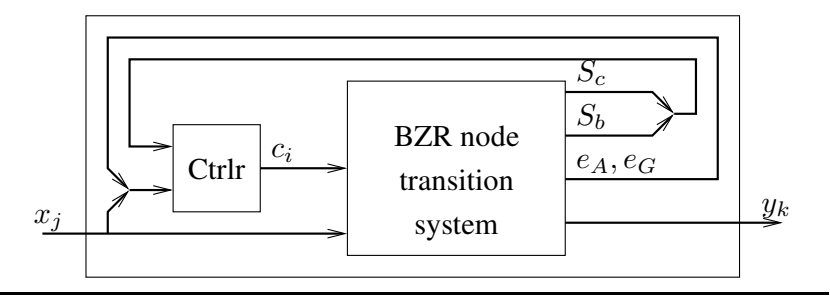

Figure 10. BZR node as DCS problem

as illustrated in Figure 11. Sub-nodes may communicate. This is where modularity gets involved, and the information about contracts of the sub-nodes, which is visible at the level of the composite, will be re-used for the compilation of the composite node.

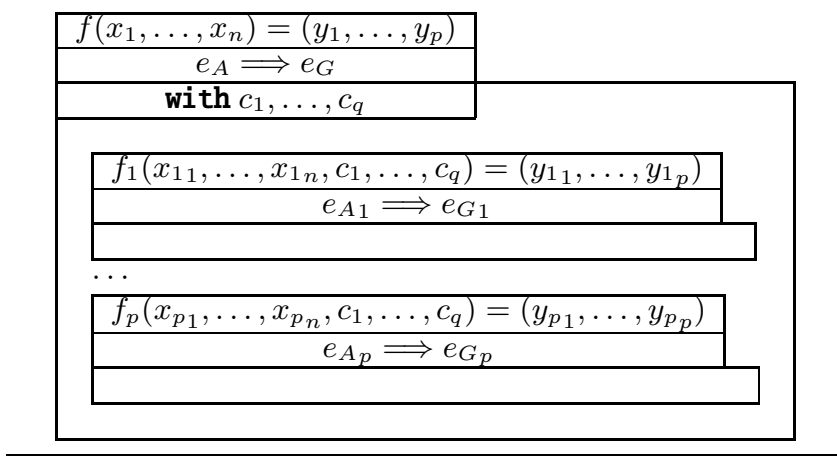

Figure 11. BZR composite node

The objective is still to control the body, using controllable variables $c_{1}, \ldots, c_{q}$, so that $e_{G}$ is true, assuming that $e_{A}$ is true. Here, we have information on sub-nodes, so that we can assume not only $e_{A}$, but also, in the case of two sub-nodes, $\left(e_{A_{1}} \Rightarrow e_{G_{1}}\right)$ and $\left(e_{A_{2}} \Rightarrow e_{G_{2}}\right)$. Accordingly, the problem becomes that: assuming $e_{A},\left(e_{A 1} \Rightarrow e_{G 1}\right)$ and $\left(e_{A 2} \Rightarrow e_{G 2}\right)$, we want to enforce $e_{A 1}$, $e_{A_{2}}$ and $e_{G}$. In particular, control at composite level takes care of enforcing assumptions of the sub-nodes.

Corresponding DCS problem The control objective is to make invariant the subset of states where $e_{A} \Rightarrow e_{G} \wedge e_{A 1} \wedge e_{A 2}$ is true. This objective is applied on the global transition system composing the contract and the body of the node, as well as the contracts for each of the sub-nodes. Note that the bodies of the sub-nodes are not used for the controller computation. Instead, we use the contracts as an abstraction of these sub-nodes. This is to partially avoid the classical state space explosion occurring when computing the whole system.

Figure 12 illustrates this; only the state variables of contracts of sub-nodes are used by the controller of the upper-level node.

\subsection{Example: Multi-task System}

We now illustrate the previous section through a simple example of a multi-task system. This example is shown for readability purpose with an ad-hoc graphical syntax; the whole final example in concrete textual syntax can be seen in appendix A.

We first specify the controller for one task, and then build a $n$ tasks server. We then illustrate how the composition of two $n$-tasks servers in order to build a $2 n$-tasks server involves introducing controllability, without which solutions cannot be found by DCS. For this, we voluntarily show pedagogic examples where the control cannot be found, before fixing them into a fully working program. 


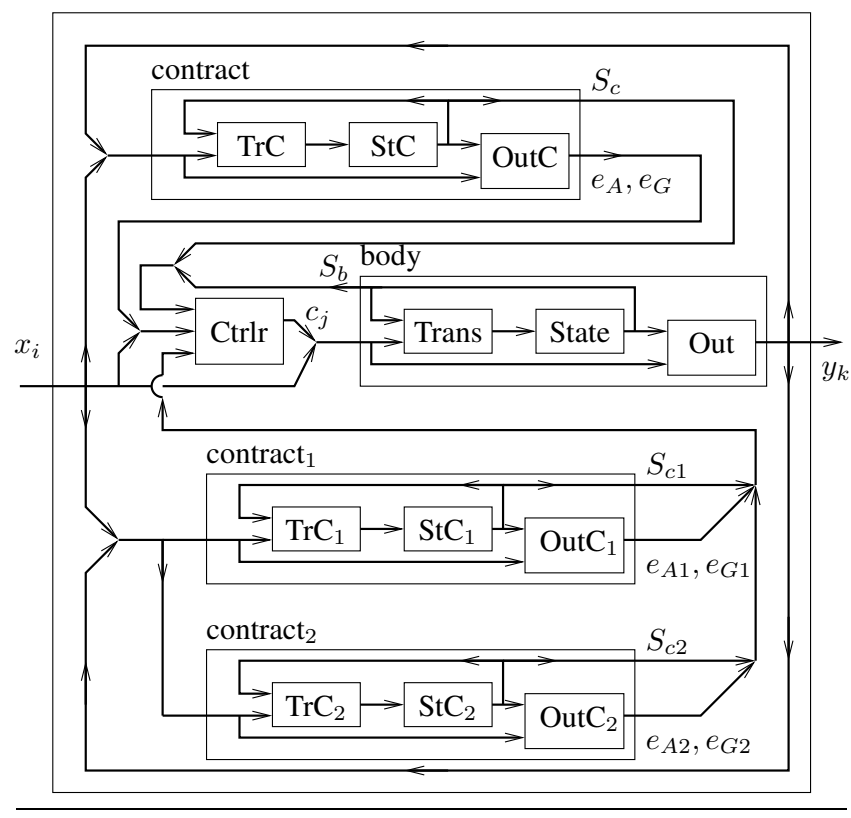

Figure 12. BZR composite DCS problem

\subsubsection{Delayable Tasks}

Figure 13 shows the control of a delayable task specified in mode automata. A delayable task takes three inputs $r, c$ and e: $r$ is the task launch request from the environment, e is the end request, and $\mathrm{c}$ is meant to be a controllable input controlling whether, on request, the task is actually launched (and therefore goes in the active state), or delayed (and then forced by the controller to go in the waiting state by stating the false value to $\mathrm{c}$ ). This node outputs a Boolean act which is true when the task is active.

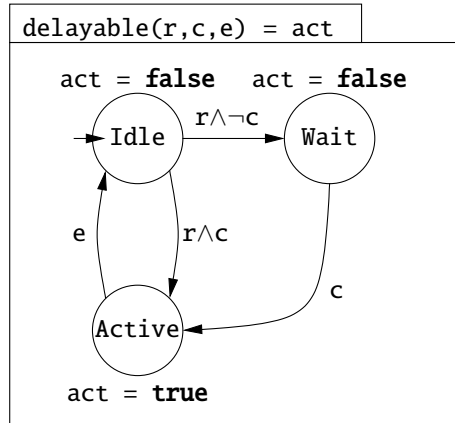

Figure 13. Delayable task (graphical syntax)

The Figure 14 shows a node ntasks where $n$ delayable tasks have been put in parallel. The tasks are inlined i.e., their code is expanded at the location of the call, so as to be able to perform DCS on this node, taking into account the tasks' states. Until now, the only interest of modularity is, from the programmer's point of view, to be able to give once the delayable task code.

This ntasks node is provided with a contract, stating that its composing tasks are exclusive, i.e., that there are no two tasks in the active state at the same instant. This is encoded in the equations defining conflicts: $c a_{i}$ is true if task $i$ is active and some other task $j, i<j \leq n$ is active too. This contract is enforced with the help of the controllable inputs $c_{i}$. Typically, the expected behavior of the obtained controller is to force to the false value the $c_{i}$ variables, when the task $i$ is requested while another task is in the active state.

\begin{tabular}{|c|}
\hline $\begin{array}{r}\text { ntasks }\left(\mathrm{c}, \mathrm{r}_{1}, \ldots, \mathrm{r}_{n}, \mathrm{e}_{1}, \ldots, \mathrm{e}_{n}\right) \\
=\left(\mathrm{a}_{1}, \ldots, \mathrm{a}_{n}\right)\end{array}$ \\
\hline $\begin{array}{c}\mathrm{ca_{1 }}=\mathrm{a}_{1} \wedge\left(\mathrm{a}_{2} \vee \ldots \vee \mathrm{a}_{n}\right) \\
\vdots \\
\mathrm{ca}_{n-1}=\mathrm{a}_{n-1} \wedge \mathrm{a}_{n}\end{array}$ \\
$\quad \begin{array}{c}\text { true } \\
\Longrightarrow\left(\mathrm{ca}_{1} \vee \ldots \vee \mathrm{ca}_{n-1}\right)\end{array}$ \\
\hline with $\mathrm{c}_{1}, \ldots, \mathrm{c}_{n}$ \\
$\mathrm{a}_{1}=$ inlined delayable $\left(\mathrm{r}_{1}, \mathrm{c}_{1}, \mathrm{e}_{1}\right)$ \\
$\vdots$ \\
$\mathrm{a}_{n}=$ inlined delayable $\left(\mathrm{r}_{n}, \mathrm{c}_{n}, \mathrm{e}_{n}\right)$ \\
\hline
\end{tabular}

Figure 14. ntasks node: $n$ delayable tasks in parallel

\subsubsection{Contract composition}

We now reuse the ntasks node, to build modularly a system composed of $2 n$ tasks. Figure 15 shows the parallel composition of two ntasks nodes. We associate to this composition a new contract, which role is to enforce the exclusivity of the $2 n$ tasks.

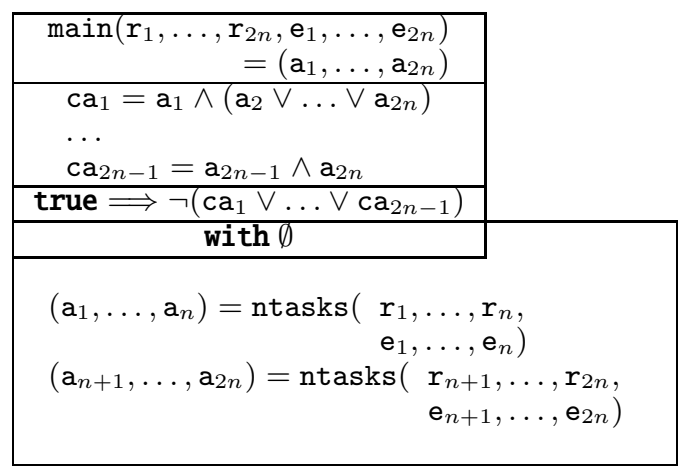

Figure 15. Composition of two ntasks nodes

It is easy to see that the contract of ntasks is not precise enough to be able to compose several of these nodes, because there is no way to control them to avoid that one task is active in each of the two subsystems. Therefore, we need to refine this contract by adding some way to externally control the activity of the tasks.

\subsubsection{Contract refinement}

We first add an input c, meant to be controllable at an upper level. The refined contract will enforce that:

1. the tasks are exclusive, i.e., that there are no two tasks in the active state at the same instant;

2. one task is active only at instants when the input $c$ is true. This property, appearing in the contract, allows a node instantiating ntasks to forbid any activity of the $n$ tasks instantiated.

The Figure 16 contains this new ntasks node.

However, the controllability introduced here is now too strong. The synthesis will succeed, but the computed controller, without knowing how c will be instantiated, will actually block all tasks in their idle state. Indeed, if the controller allows one task to go in its active state, the input c (uncontrollable at the ntasks level) can become false at the next instant, violating the property to enforce. 




Figure 16. First contract refinement for the ntasks node

Thus, we propose to add an assumption to this contract: the input c will not become false if a task was active an instant before. This assumption will be enforced correct by the controller of the upper level. This new contract is visible in Figure 17.

\begin{tabular}{|c|}
\hline $\begin{array}{r}\operatorname{ntasks}\left(\mathrm{c}, \mathrm{r}_{1}, \ldots, \mathrm{r}_{n}, \mathrm{e}_{1}, \ldots, \mathrm{e}_{n}\right) \\
=\left(\mathrm{a}_{1}, \ldots, \mathrm{a}_{n}\right)\end{array}$ \\
\hline $\begin{array}{l}\mathrm{ca}_{1}=\mathrm{a}_{1} \wedge\left(\mathrm{a}_{2} \vee \ldots \vee \mathrm{a}_{n}\right) \\
\ldots \\
\mathrm{ca} \mathrm{a}_{n-1}=\mathrm{a}_{n-1} \wedge \mathrm{a}_{n} \\
\text { one }=\mathrm{a}_{1} \vee \ldots \vee \mathrm{a}_{n} \\
\text { pone }=\text { false fby one }\end{array}$ \\
\hline $\begin{array}{c}(\neg \text { pone } \vee c) \\
\Longrightarrow \neg\left(\mathrm{ca}_{1} \vee \ldots \vee \mathrm{ca}_{n-1}\right) \wedge(\mathrm{c} \vee \neg \text { one })\end{array}$ \\
\hline with $\mathrm{c}_{1}, \ldots, \mathrm{c}_{n}$ \\
\hline $\begin{array}{l}\mathrm{a}_{1}=\text { inlined delayable }\left(r_{1}, c_{1}, e_{1}\right) \\
\cdots \\
a_{n}=\text { inlined delayable }\left(r_{n}, c_{n}, e_{n}\right)\end{array}$ \\
\hline
\end{tabular}

Figure 17. Second contract refinement for the ntasks node

We can then use this new ntasks version for the parallel composition, by instantiating the $\mathrm{c}$ input by two controllable variables. This composition can be found in Figure 18 .

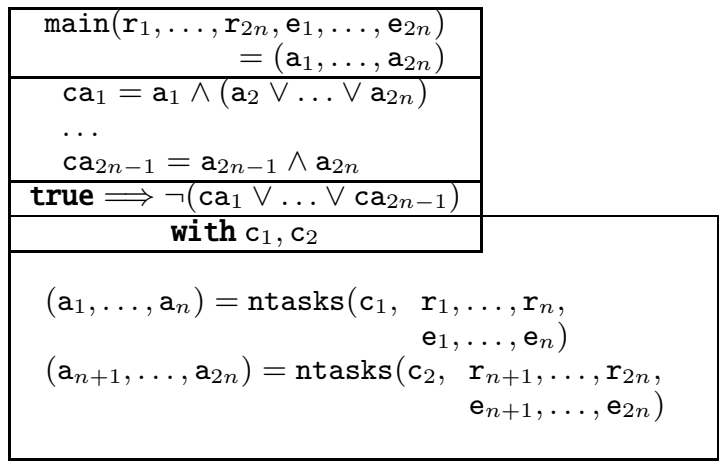

Figure 18. Two refined ntasks parallel composition

\section{BZR compilation}

This section describes in a formal way the compilation process of our language. This compilation process is modular, meaning that each node will be compiled in an independent way; and it comprises a discrete controller synthesis stage for each of these nodes. We first recall the notations used, then present the controller synthesis for one contract, and last show how the synthesized controllers are recombined to obtain the whole controlled system.

\subsection{Control of Symbolic Transition Systems (STS)}

\subsubsection{Definitions}

Notations. Given a set of Boolean variables $Z=Z_{1}, \cdots, Z_{k}$, we define a valuation of $Z$ as a function $v a l: Z \rightarrow \mathbb{B}^{k}$ that assigns to each variables in $Z$ a value either true or false. In the sequel, we shall use $X, Y, Z$ as vectors of Boolean variables and $x, y, z$ for a possible valuation of these vectors. Given a predicate $B \in \mathbb{B}[Z]$ a polynomial over $Z$ and $z \in \operatorname{val}(Z)$, we denote by $B(z) \in \mathbb{B}$ the predicate $B$ valuated by $z$. We further denote $\bar{z}=z_{0} . z_{1} . z_{2} \ldots$ an infinite sequence of valuations of $Z$. Given a sequence $\bar{z}$ and a predicate $G \in \mathbb{B}[Z]$, we denote $\bar{z} \models \square G$ the fact that $G$ hold for the sequence $\bar{z}$ at every instant.

$$
\bar{z} \models \square G \text { iff } \bar{z}=z_{0} \cdot z_{1} \cdot z_{2} \cdots \text { and } \forall i, G\left(z_{i}\right) .
$$

Symbolic Transition Systems. We represent synchronous programs by Synchronous Symbolic Transition Systems (STS). A STS $S(X, Y, Z)$, defining a synchronous program of state variables $X \in \mathbb{B}^{m}$, input event variables $Y \in \mathbb{B}^{n}$, output event variables $Z \in \mathbb{B}^{p}$, is a tuple $\left(P, O, Q, Q_{0}\right)$ :

$$
S=\left\{\begin{array}{l}
X^{\prime}=P(X, Y) \\
Z=O(X, Y) \\
Q(X, Y) \\
Q_{0}(X)
\end{array}\right.
$$

where:

- the vectors $X$ and $X^{\prime}$ respectively encode the current and next states of the system and are called state variables (they contain the memory necessary for describing the system behavior).

- $P \in \mathbb{B}[X, Y]$ represent the transition function. It is a vectorvalued function $\left[P_{1}, \ldots, P_{n}\right]$ from $\mathbb{B}^{n+m}$ to $\mathbb{B}^{n}$. Each polynomial component $P_{i}$ represents the evolution of the state variable $X_{i}$. It characterizes the dynamic of the system. between the current state $X$ and events $Y$ and next state $X^{\prime}$.

- $O \in \mathbb{B}[X, Y]$ represents the output function.

- $Q_{0} \in \mathbb{B}[X]$ defines the set initial states, and $Q \in \mathbb{B}[X, Y, Z]$ the constraints between current states and events and encodes the static part of the system (invariant for all instants $t$ ).

The semantics of a STS $S$ is that it defines a set of sequences $\overline{(x, y, z)}$ such that : $Q_{0}\left(x_{0}\right)$ and $\forall i$,

$$
Q\left(x_{i}, x_{i}\right) \wedge\left(x_{i+1}=P\left(x_{i}, x_{i}\right)\right) \wedge\left(z_{i}=O\left(x_{i}, y_{i}\right)\right) .
$$

We denote by $\operatorname{Traces}(S)$ this set of sequences.

STS transformations. Given two STS $S_{1}$ and $S_{2}$, we note by $S_{1} \| S_{2}$, the synchronous parallel composition of $S_{1}$ and $S_{2}$ which consists in performing the conjunction of the composing predicate of $S_{1}$ and $S_{2}$, and is defined iff state and output variables are exclusive. Communications between the two systems are expressed via common inputs and outputs variables, which are considered as outputs of the composition. Formally, $S_{1} \| S_{2}$ is a STS $S_{1} \| S_{2}\left(X_{1} \cup\right.$ 


$$
\begin{aligned}
& X_{2},\left(Y_{1} \cup Y_{2}\right) \backslash(\left.\left.Z_{1} \cup Z_{2}\right), Z_{1} \cup Z_{2}\right): \\
& S_{1} \| S_{2}=\left\{\begin{array}{l}
X_{1}^{\prime}, X_{2}^{\prime}=P_{1}\left(X_{1}, Y_{1}\right) \wedge P_{2}\left(X_{2}, Y_{2}\right) \\
Z_{1}, Z_{2}=\left(O_{1}\left(X_{1}, Y_{1}\right), O_{2}\left(X_{2}, Y_{2}\right)\right) \\
Q_{1}\left(X_{1}, Y_{1}\right) \wedge Q_{2}\left(X_{2}, Y_{2}\right) \\
Q_{01}\left(X_{1}\right) \wedge Q_{02}\left(X_{2}\right)
\end{array}\right.
\end{aligned}
$$

Finally, we denote by $S \triangleright A$ the extension of constraints of $S$ with the predicate $A \in \mathbb{B}[X, Y, Z]$ :

$$
S \triangleright A=\left\{\begin{array}{l}
X^{\prime}=P(X, Y) \\
Z=O(X, Y) \\
Q(X, Y, Z) \wedge A(X, Y, Z) \\
Q_{0}(X)
\end{array}\right.
$$

Contracts satisfaction. In the sequel, we shall consider properties expressed by means of contracts that are defined as follows:

Definition 1 (Contract). A contract is a tuple $C=\left(S^{c}, A, G\right)$ where $S^{c}\left(X^{c},(Y \cup Z), \emptyset\right)$ is a STS, $A \in \mathbb{B}\left[X^{c}\right]$ and $G \in \mathbb{B}\left[X^{c}\right]$ are predicates.

Intuitively, $S^{c}$ can be seen as an abstraction of a component program, $G$ is the property to be satisfied by the traces of the component on which this contract is placed providing the fact that the model of the environment $A$ is satisfied. For clarity, we define the contract predicates $A$ and $G$ on only state variables of the contract. We remark though that this does not restrict the expressiveness of these properties, as one can add dummy state variables constrained with inputs or outputs values, so as to be able to express properties upon inputs and outputs variables.

Definition 2 (Contract fulfilment). A STS $S(X, Y)$ fulfills a contract $C=\left(S^{c}, A, G\right)$, noted $S \models C$, if $\forall \overline{\left(x, x^{c}, y, z\right)} \in$ $\operatorname{Traces}\left(S \| S^{c}\right), \overline{\left(x^{c}\right)} \models \square A \Rightarrow \overline{\left(x^{c}\right)} \models \square G$.

Hence, a contract is satisfied whenever the traces of $S$, composed with $S^{c}$ and satisfying $\square A$, satisfy $\square G$. This constitutes one of the main difference with the work of [6], as in their framework, $A$ and $G$ are assertions of any kind on traces. Our restriction is due to the technique used: only safety properties (vs liveness or equity) can be ensured by abstracted state space exploration, and preserved by synchronous composition.

As the above definition does not allow to be easily applied on STS, we give below a property on contracts: the environment model can be viewed as additional constraints of the STS composed of $S$ and the contract program $S^{c}$.

Proposition 1. $\left(S \| S^{c}\right) \triangleright A \models G \Rightarrow S \models\left(S^{c}, A, G\right)$.

\subsubsection{Contracts enforcement}

Assume given a system $S$ and a contract $C$ on $S$. Our aim is to restrict the behavior of $S$ in order to fulfil the contract.

The control of a STS relies on a distinction between events. We distinguish between the uncontrollable event variables $Y^{u c}$ which are defined by the environment, and the controllable event $Y^{c}$ which are defined by the controller of the system (they are considered as internal variables). Now, in order to enforce $C=$ $\left(S^{c}, A, G\right)$ with $S^{c}\left(X^{c}, Y^{u c} \cup Z, \emptyset\right), A \in \mathbb{B}\left[X^{c}\right]$ and $G \in \mathbb{B}\left[X^{c}\right]$ on $S$ we consider the STS $\left(S \| S^{c}\right) \triangleright A$ :

$$
\left(S \| S^{c}\right) \triangleright A=\left\{\begin{array}{l}
X^{\prime}, X^{\prime c}=P\left(X, X^{c}, Y^{c}, Y^{u c}\right) \\
Z=O\left(X, X^{c}, Y^{c}, Y^{u c}\right) \\
Q\left(X, X^{c}, Y^{c}, Y^{u c}\right) \\
Q_{0}\left(X, X^{c}\right)
\end{array}\right.
$$

The property we wish to enforce by control on this system is given by the invariant $G$. In our framework, a controller is a predicate $K \in \mathbb{B}\left[X, X^{c}, Y^{c}, Y^{u c}\right]$ that constraints the set of admissible event so that the traces of the controlled system always satisfy the predicate $G$. We do not detail here how such a controller can be computed. It relies on a fix-point computation w.r.t. the function $\operatorname{Pre}_{u c}(E)=\left\{\left(x, x^{c}\right) \mid \forall y^{u c}, \exists y^{c}, Q\left(x, y^{u c}, y^{c}\right) \Rightarrow\right.$ $\left.P\left(x, y^{u c}, y^{c}\right) \in E\right\}$. We will present a more generic algorithm in the next section. The controller describes how to choose the static controls; when the controlled system is in state $\left(x, x^{c}\right)$, and when an event $y^{u c}$ occurs, any value $y^{c}$ such that $Q\left(x, x^{c}, y^{u c}, y^{c}\right)$ and $K\left(x, x^{c}, y^{u c}, y^{c}\right)$ can be chosen. The behavior of the system supervised by the controller is then modeled by $\left(S \| S^{c}\right) \triangleright K$.

Determination of the controller. Assume now that we have computed a controller $K \in \mathbb{B}\left[X, X^{c}, Y^{c}, Y^{u c}\right]$. $K$ is non-deterministic w.r.t. the controllable variables, in the sense that for each state of the system and for each valuation of the uncontrollable variables, there might exists several valuations for the controllable ones that respects $K$. Obviously, this non-determinism has to be solved in some ways. One possibility is to encapsulate in the system, a predicate solver, that either asks an external user to make a choice amongst the possible solutions or that itself performed a random choices amongst them. Following a method similar to the one described in [13], another possibility is to derive from the controller a set of functions $F_{i}^{c}$ that depends on $X, X^{c}, Y^{u c}$ and some fresh phantom variables $\phi_{i}$, one for each controllable variables, namely:

$$
\begin{aligned}
& K\left(X, X^{c}, Y^{c}, Y^{u c}\right) \Leftrightarrow \\
& \exists\left(\phi_{i}\right)_{i \leq \ell}\left\{\begin{array}{l}
Y_{1}^{c}=F_{1}^{c}\left(X, X^{c}, Y^{u c}, \phi_{1}\right) \\
\cdots \\
Y_{i}^{c}=F_{i}^{c}\left(X, X^{c}, Y^{u c}, Y_{1}^{c}, \cdots, Y_{i-1}^{c}, \phi_{i}\right) \\
\cdots \\
Y_{\ell}^{c}=F_{n}^{c}\left(X, X^{c}, Y^{u c}, Y_{1}^{c}, \cdots, Y_{\ell-1}^{c}, \phi_{\ell}\right) \\
K^{\prime}\left(X, X^{c}, Y^{u c}\right)
\end{array}\right.
\end{aligned}
$$

In other words, whatever the valuation of a tuple $\left(x, x^{c}, y^{u c}, y^{c}\right)$, there exists a valuation $\left(v_{\phi_{i}}\right)_{i \leq \ell}$ of $\left(\phi_{i}\right)_{i \leq \ell}$ such that $y_{i}^{c}=$ $F_{i}^{c}\left(x, x^{c}, y^{u c}, v_{\phi_{i}}\right)$.

At this point, either the variables $\left(\phi_{i}\right)$ can be seen as new inputs of the system or can be eliminated by choosing for each of them a value. Note that in this case, we loose the equivalence (only $\Rightarrow$ implication is kept). For clarity reasons, this is the second choice we have made in this paper.

Remark 1. With the determination of the controller, part of the solutions can be actually lost, when the synthesized controller is not deterministic (but the safety property is kept); and in this sense the modular control is sub-optimal. It is an interesting perspective indeed to replace local random selections with a heuristic taking into account some of the interactions between components. Note however, that it is possible to keep the maximal behavior by keeping the new phantom variables.

\subsection{Modular control of STS}

\subsubsection{Contracts composition}

Let consider now a hierarchically designed program, i.e., a STS $S(X, Y, Z)$ composed of subcomponents $S_{1}, \ldots, S_{n}$, together with additional local code $S^{\prime}$ (as in Figure 19). We have then :

$$
S(X, Y, Z)=\left(S^{\prime}\left\|S_{1}\right\| \ldots \| S_{n}\right)
$$

Note that $Y_{i}^{u c} \subseteq X \cup Y^{u c} \cup Y^{c} \cup Z$, namely the uncontrollable variables of the lower level can be defined either by state, uncontrollable or controllable inputs, or outputs variables of the upper system. Thus to proceed to the encapsulation we need to rename the variables $Y_{i}^{u c}$ according to their new name in the new system.

We assume that each sub-component $S_{i}\left(X_{i}, Y_{i}, Z_{i}\right)$ comes with a contract $C_{i}=\left(S_{i}^{c}, A_{i}, G_{i}\right)$, with $S_{i}^{c}\left(X_{i}^{c}, Y_{i} \cup Z_{i}, \emptyset\right), A_{i} \in$ $\mathbb{B}\left[X_{i}^{c}\right], G_{i} \in \mathbb{B}\left[X_{i}^{c}\right]$, and that a controller $K_{i}$ has been computed such as, for all $i,\left(S_{i} \| S_{i}^{c}\right) \triangleright K_{i} \models C_{i}$. 


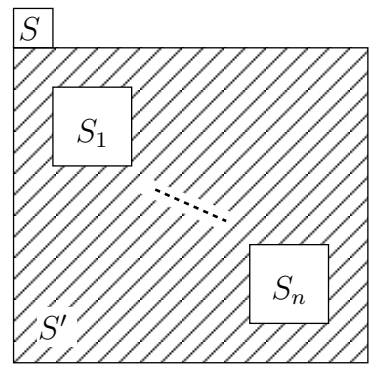

Figure 19. STS composed of several subcomponents

We want now to obtain a controller $K$ for the system $S$ to fulfill a contract $C=\left(S^{c}, A, G\right)$, with $S^{c}\left(X^{c}, Y \cup Z, \emptyset\right), A \in \mathbb{B}\left[X^{c}\right]$ and $G \in \mathbb{B}\left[X^{c}\right]$. One way to do this is to compute the whole dynamic of $S$ and to control it using the previous method, but this would lead to a state space explosion. Instead, we will use the contracts of the sub-components as an abstraction of them. Thus, we use an abstracted STS $\hat{S}$, defined as the composition of $S^{\prime}$ with the system part of the subcontracts, constrained with the properties enforced by $K_{i}$ on each of the sub-components. In other words, we take the assume and enforced parts of the subcontracts as environment model of the abstracted system. Moreover, the $Z_{i}$ variables were outputs of the lower level. As we abstract away the body of this system, these variables have now to be considered as uncontrollable variables of the upper system (indeed, there is no way to know their value). Besides, the value of these variables is normally computed according to the value of $Y_{1}^{u c}$ and internal variables. Hence there exists causality problems between these variables and the variables of the upper level. This causality constraint will be resolved by the design of a more general controller synthesis algorithm (see Section 4.2.2). We define the new system to be controlled as follows:

$$
\begin{aligned}
& \hat{S}\left(X, Y^{u c} \cup Z_{1} \cup \ldots \cup Z_{n}, Y^{c}, Z\right)= \\
& \quad\left(S^{\prime}\left\|\left(S_{1}^{c} \triangleright\left(A_{1} \Rightarrow G_{1}\right)\right)\right\| \ldots \|\left(S_{n}^{c} \triangleright\left(A_{n} \Rightarrow G_{n}\right)\right)\right)
\end{aligned}
$$

We should notice that, in order to the STSs $S_{i}$, controlled by their controller, to be evaluated in a correct environment, the predicates $A_{i}$ must be satisfied. Therefore, we define a new contract $\hat{C}$, which will be used to compute a controller on $\hat{S}$ :

$$
\hat{C}=\left(S^{c}, \hat{A}, \hat{G}\right) \text { where }\left\{\begin{array}{l}
\hat{A}=A \\
\hat{G}=G \wedge A_{1} \wedge \ldots \wedge A_{n}
\end{array}\right.
$$

We then compute controller $K$, enforcing contract $C$ on STS $\hat{S}$ :

$$
\hat{S} \triangleright K \models \hat{C}
$$

The correction of this method is given by the following result, stating that the controller $K$, computed for the abstracted system $\hat{S}$, is a correct controller for the concrete system, i.e., $S$ controlled by $K$ fulfils the contract $C$.

\section{Theorem 1.}

If $\hat{S} \triangleright K \models \hat{C}$ and for all $i,\left(S_{i} \| S_{i}^{c}\right) \triangleright K_{i} \models C_{i}$, then:

$$
\left(S^{\prime}\left\|\left(S_{1} \| S_{1}^{c}\right) \triangleright K_{1}\right\| \ldots \|\left(S_{n} \| S_{n}^{c}\right) \triangleright K_{n}\right) \triangleright K \models C
$$

\subsubsection{Control of an STS with sub-contracts}

As mentioned in the previous section, there exists causality dependencies between the $Z_{i}$ variables and the $Y_{i}^{c}$ variables, in the sense that the computation of the value of the variables $Z_{i}$ depends on the value of $Y_{i}^{c}$, which must then be computed before. Moreover, the $Y_{i}^{c}$ can be computed according to the value of some other variables of the upper-level system. In the following, we denote by $X_{1} \prec X_{2}$ the fact that $X_{2}$ depends on $X_{1}$. This relation is straightforwardly extended to variable sets:

$$
\tilde{X} \prec \tilde{Y} \stackrel{\text { def }}{=} \forall(X, Y) \in \tilde{X} \times \tilde{Y}, X \prec Y
$$

In the sequel, we shall consider the following subsets of $Z_{i}$ : $\left(\tilde{Z}_{i}\right)_{i \leq n}$, where $\forall i \leq n, \tilde{Z}_{i} \subseteq \tilde{Z}_{i-1}$, and such that $Y_{i}^{c} \prec \tilde{Z}_{i}$. Furthermore, we note $\tilde{Z}_{i}^{\prime}=\tilde{Z}_{i} \backslash \tilde{Z}_{i-1}$.

$$
Y \prec \tilde{Z}_{0} \prec\left\{Y_{1}^{c}\right\} \prec \tilde{Z}_{1} \prec \cdots \prec\left\{Y_{n}^{c}\right\} \prec \tilde{Z}_{n}
$$

Control synthesis algorithm overview. Computing the controller $K$ as in Section 4.1.2 is not suitable as it does not take into account the dependencies between the variables. Intuitively, in order to be able to compute the value of $Y_{i}^{c}$ we need to know the value of the variables $\tilde{Z}_{i}$. Thus, if we want to be sure that there exists a solution to the determination, the controller should take into account that the value of the variable $Y_{i}^{c}$ is correct whatever the value of $\tilde{Z}_{i}$. This is what we capture by computing the $\operatorname{Pre}_{u c}$ operator as follows:

$$
\begin{array}{r}
\operatorname{Pre}_{u c}(E)=\left\{\left(x, x^{c}\right) \mid \forall y^{u c}, \tilde{z}_{0}, \exists y_{1}^{c}, \forall \tilde{z}_{1}^{\prime}, \ldots, \exists y_{n}^{c}, \forall \tilde{z}_{n}^{\prime},\right. \\
\left.Q\left(x, y^{u c}, y^{c}, z\right) \Rightarrow P\left(x, y^{u c}, y^{c}, z\right) \in E\right\}
\end{array}
$$

Further the computation of $K$ is similar to the one of Section 4.1.2.

Deterministic controller. For the determination of $K$, we also need to take into account the dependencies between the variables: the order relation (1) implies that the deterministic controller, as defined in Section 4.1.2, will be a set of functions $\tilde{F}_{i}^{c}$ that depends on $X, X^{c}, Y^{u c}, \phi_{i}$ and the additional uncontrollable inputs $\tilde{Z}_{i-1}$ :

$$
\begin{gathered}
K\left(X, X^{c}, Y^{c}, Y^{u c}\right) \\
\Leftrightarrow\left(\phi_{1}, \cdots, \phi_{l}\right), \\
\left\{\begin{array}{l}
Y_{1}^{c}=\tilde{F}_{1}^{c}\left(X, X^{c}, Y^{u c}, \tilde{Z}_{0}, \phi_{1}\right) \\
\cdots \\
Y_{i}^{c}=\tilde{F}_{i}^{c}\left(X, X^{c}, Y^{u c}, Y_{1}^{c}, \cdots, Y_{i-1}^{c}, \tilde{Z}_{i-1}, \phi_{i}\right) \\
\cdots \\
Y_{n}^{c}=\tilde{F}_{n}^{c}\left(X, X^{c}, Y^{u c}, Y_{1}^{c}, \cdots, Y_{n-1}^{c}, \tilde{Z}_{n-1}, \phi_{l}\right)
\end{array}\right.
\end{gathered}
$$

These $\tilde{F}_{i}^{c}$ functions are related with $F_{i}^{c}$, defined in Section 4.1.2, by $\tilde{F}_{i}^{c}=\forall \tilde{Z}_{i}, F_{i}^{c}$. The existence of $\tilde{F}_{i}^{c}$ is then ensured by the modified version of the control synthesis algorithm we give further. Once again, we can further eliminate the variables $\left(\phi_{i}\right)$ by choosing a particular value for each of them.

\section{Implementation}

Figure 20 illustrates the compilation process for BZR implementing our method. Boxes with round corners indicate data (source code, target code, intermediate formats); rectangular boxes indicate tools and operations. It is built around synchronous compilation and DCS technology, and borrows essentially two pre-existing tools (indicated in stripped boxes). One is a synchronous compiler: HEPTAGON, used in order to (i): compile the nodes into a format accepted by the DCS tool, and (ii): compile the composition of the triangularized controller with the originally uncontrolled automaton, hence building the controlled automaton, and generating executable code for it. The other is the DCS tool: SIGALI. BZR has been used for a case study of a video display on a mobile phone [2].

Our approach is target independent, in the sense that the compilation process from automata to code generation concerns the transition function of the controller. The target is taken into account, as shown in the development process of Figure 2, when the control part is extracted from the target, and when the resulting step function is linked back into the executive, with the proper interface. The general structure of the generated code consists of two functions: 


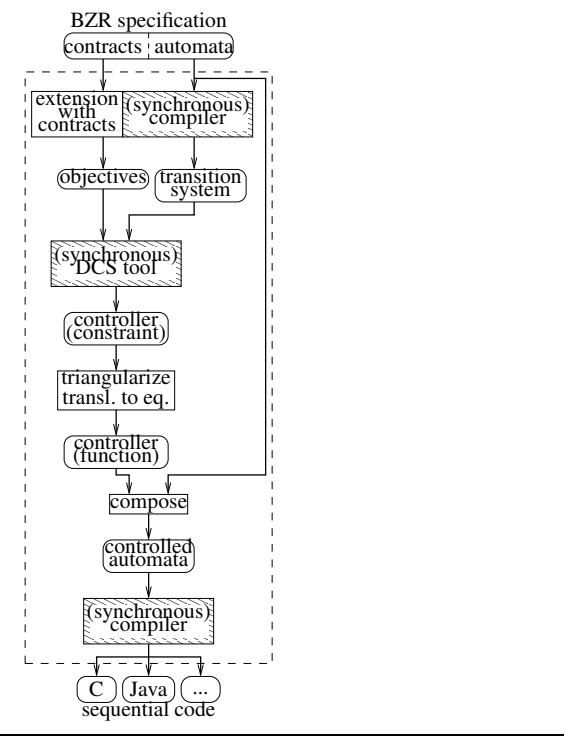

Figure 20. BZR compilation process.

a step function, performing one transition, with input events as parameters, producing output values, and updating the internal state of the automaton; and a reset function, for initialization purposes. These functions (signature and body) are simple enough to allow to target any general-purpose language. The compiler currently generates $\mathrm{C}$ or Java code. It would not be difficult to consider also more domain-specific languages like VHDL.

\section{Performances}

The bottleneck of our approach is clearly the synthesis time, as the algorithm is based on the exploration of the state space of each node. This section thus shows some typical synthesis time on multitasks systems, with and without use of modularity.

The system considered here is composed of $3 n$ tasks, to be enforced exclusive, as showed in Section 3.2. These $3 n$ tasks are composed of $n$ delayable tasks (Figure 13) and $2 n$ rejectable tasks (Figure 21: tasks requests can be rejected but are not memorized). Three experiments are made:

1. the $3 n$ tasks are inlined into the same node: the state space to explore corresponds then to the entire state space of the $3 n$ automata composition;

2. the $3 n$ tasks are decomposed into three nodes instantiation of $n$ inlined tasks, as in Section 3.2: three separate synthesis are then performed (one for $n$ delayable tasks, one for $n$ rejectable tasks and one for the main node);

3. likewise, the $3 n$ tasks are decomposed into three nodes, but we also use the modularity to perform the three synthesis in parallel, as they do not depend on each other's results.

Figure 22 shows the compared synthesis time for each of these experiments. They have been fulfilled on a standard PC with two $2.33 \mathrm{GHz}$ cores, and $4 \mathrm{~Gb}$ of RAM. The non-monotony of the two curves are explained by the sensitivity of the underlying algorithms, which makes the synthesis time hard to predict. Nevertheless, these measurements shows that modularity can improve the usability of discrete controller synthesis. We can see, e.g., that while synthesis fail (for lack of memory) in "inlined" mode for $n=14,15$ and $n>$ 16 , such systems when adequately structured can be handled in few seconds. Moreover, modularity allows to gain some synthesis time by parallelizing the synthesis computations.

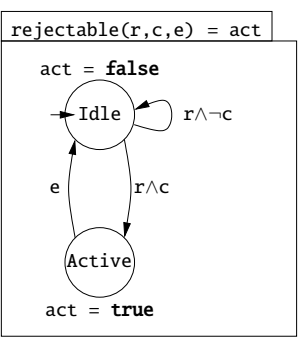

Figure 21. Rejectable task

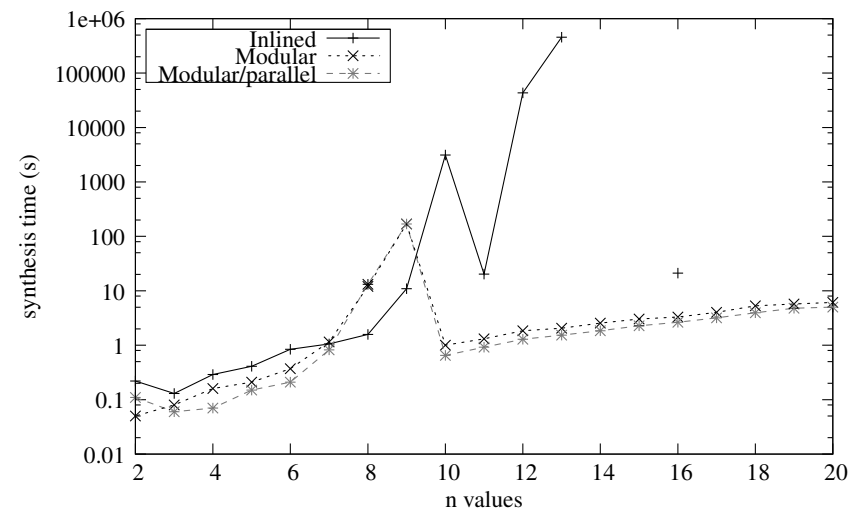

Figure 22. Compared synthesis time for $3 n$ automata in inlined, modular and modular+parallel modes

Concerning other performance aspects, the final controller size is, for the same reasons as the synthesis time, exponential in the size of the initial program (typically, number of states and inputs). This controller consists of sequences of conditionals (translation of binary decision diagrams), and also its online evaluation is polynomial in the size of the initial program.

\section{Conclusion}

We have proposed a programming-level method, based on the "design by contracts" principle, to apply modular discrete controller synthesis, integrated into a compilation process. This method allows to apply DCS on subcomponents of a system, in order to compute one single controller for each of these subcomponents. These controllers can then be composed with their associated components, before their composition in an upper-level component. The contracts can then be used to compute a controller for this upper-level component, abstracting the bodies of its subcomponents; and so on. On the other hand, in the comparison between modular and monolithic synthesis, we have the advantage of breaking down the cost of synthesis computations (as we only keep the guaranty at the upper level and not the current node that can be itself a composition of several nodes), for which real-size evaluations are in our projects. More precisely, our contribution is a new nondeterministic synchronous language with contracts, named BZR. We have shown how this language can be compiled towards symbolic transition systems, and how modular controllers are computed and recomposed.

Further work can be fulfilled toward several directions. This method could be applied in different component framework, so as to explore its interaction with actual industrial design flow. Some work is in progress within the Fractal framework. Such integration in actual design flow entails a greater interactivity with the programmer: thus, efforts on diagnosis should be made. Some meth- 
ods should be proposed to get intelligible informations to the programmer when the synthesis fail, which is not currently possible. Another interesting prospect would also be, when synthesis fail, to use the synthesis algorithm to infer some additional constraints on the contracts of the program to allow the synthesis to succeed.

In a more technical concern, some other controller synthesis methods or algorithms could replace the SIGALI tool. This work only addresses invariance objectives: other kinds of synthesis objective (accessibility, attractivity) would jeopardize the modularity properties, but are an interesting prospect to deal with.

\section{References}

[1] S. Abdelwahed and W. Wonham. Supervisory control of interacting discrete event systems. In 41th IEEE Conference on Decision and Control, pages 1175-1180, Las Vegas, USA, December 2002.

[2] S. Aboubekr, G. Delaval, and E. Rutten. A programming language for adaptation control: Case study. In 2nd Workshop on Adaptive and Reconfigurable Embedded Systems (APRES 2009). ACM SIGBED Review, volume 6, Grenoble, France, Oct. 2009.

[3] K. Akesson, H. Flordal, and M. Fabian. Exploiting modularity for synthesis and verification of supervisors. In Proc. of the IFAC, 2002.

[4] K. Altisen, A. Clodic, F. Maraninchi, and E. Rutten. Using controller synthesis to build property-enforcing layers. In Proceedings of the European Symposium on Programming (ESOP'03), number 2618 in LNCS, Warsaw, Poland, Apr. 2003.

[5] R.-J. Back and C. C. Seceleanu. Contracts and games in controller synthesis for discrete systems. In IEEE Int. Conf. on Engineering of Computer-Based Systems, page 307, 2004.

[6] A. Benveniste, B. Caillaud, and R. Passerone. A generic model of contracts for embedded systems. Res. Rep. RR-6214, INRIA, 2007.

[7] A. Benveniste, P. Caspi, S. Edwards, N. Halbwachs, P. L. Guernic, and R. de Simone. The synchronous languages twelve years later. Proc. of the IEEE, 91(1):64-83, Jan. 2003.

[8] C. Cassandras and S. Lafortune. Introduction to Discrete Event Systems. Kluwer Academic Publishers, 1999.

[9] A. Chakrabarti, L. de Alfaro, T. Henzinger, and F. Mang. Synchronous and bidirectional component interfaces. In CAV 2002: 14th Int. Conf. on Computer Aided Verification, LNCS, 2002.

[10] J.-L. Colaço, B. Pagano, and M. Pouzet. A Conservative Extension of Synchronous Data-flow with State Machines. In ACM Int. Conf. on Embedded Software (EMSOFT'05), Jersey city, New Jersey, USA, September 2005.

[11] M. De Queiroz and J. Cury. Modular control of composed systems. In Proceedings of the American Control Conference, pages 4051-4055, Chicago, Illinois, June 2000.

[12] G. Delaval and E. Rutten. A domain-specific language for multi-task systems, applying discrete controller synthesis. J. on Embedded Systems, 2007(84192):17, Jan. 2007. wWW . hindawi . com/journals/es.

[13] Y. Hietter, J.-M. Roussel, and J.-J. Lesage. Algebraic Synthesis of Transition Conditions of a State Model. In Proc. of 9th Int. Workshop On Discrete Event Systems (WODES'08), Göteborg, June 2008.

[14] R. Leduc, W. Wonham, and M. Lawford. Hierarchical interface-based supervisory control: Parallel case. In Proc. of the 39th Allerton Conf. on Comm., Contr., and Comp., pages 386-395, October 2001.

[15] C. Ma and W. Wonham. A symbolic approach to the supervision of state tree structures. In 13th Mediterranean Conference on Control and Automation, Limassol, Cyprus, June 2005.

[16] F. Maraninchi and L. Morel. Logical-time contracts for the development of reactive embedded software. In 30th Euromicro Conference, Component-Based Software Engineering Track (ECBSE), Rennes, France, Sept. 2004.

[17] H. Marchand, P. Bournai, M. L. Borgne, and P. L. Guernic. Synthesis of discrete-event controllers based on the Signal environment. Discrete Event Dynamic System: Theory and Applications, 10(4), Oct. 2000 .
[18] H. Marchand and B. Gaudin. Supervisory control problems of hierarchical finite state machines. In 41th IEEE Conference on Decision and Control, Las Vegas, USA, December 2002.

[19] B. Meyer. Applying "design by contract". Computer, 25(10):40-51, Oct 1992.

[20] P. J. Ramadge and W. M. Wonham. The control of discrete event systems. Proceedings of the IEEE; Special issue on Dynamics of Discrete Event Systems, 77(1):81-98, 1989.

[21] Y. Wang, S. Lafortune, T. kelly, M. Kudlur, and S. Mahlke. The Theory of Deadlock Avoidance via Discrete Control. In ACM Symposium on Principles of Programming Languages (POPL '09), Savannah, Georgia, USA, January 2009.

[22] Y. Willner and M. Heymann. Supervisory control of concurrent discrete-event systems. Int. J. of Control, 54(5):1143-1169, 1991.

\section{A. BZR Example in Concrete Textual Syntax}

The following is the concrete BZR code (apart from indices of identifiers) for the example.

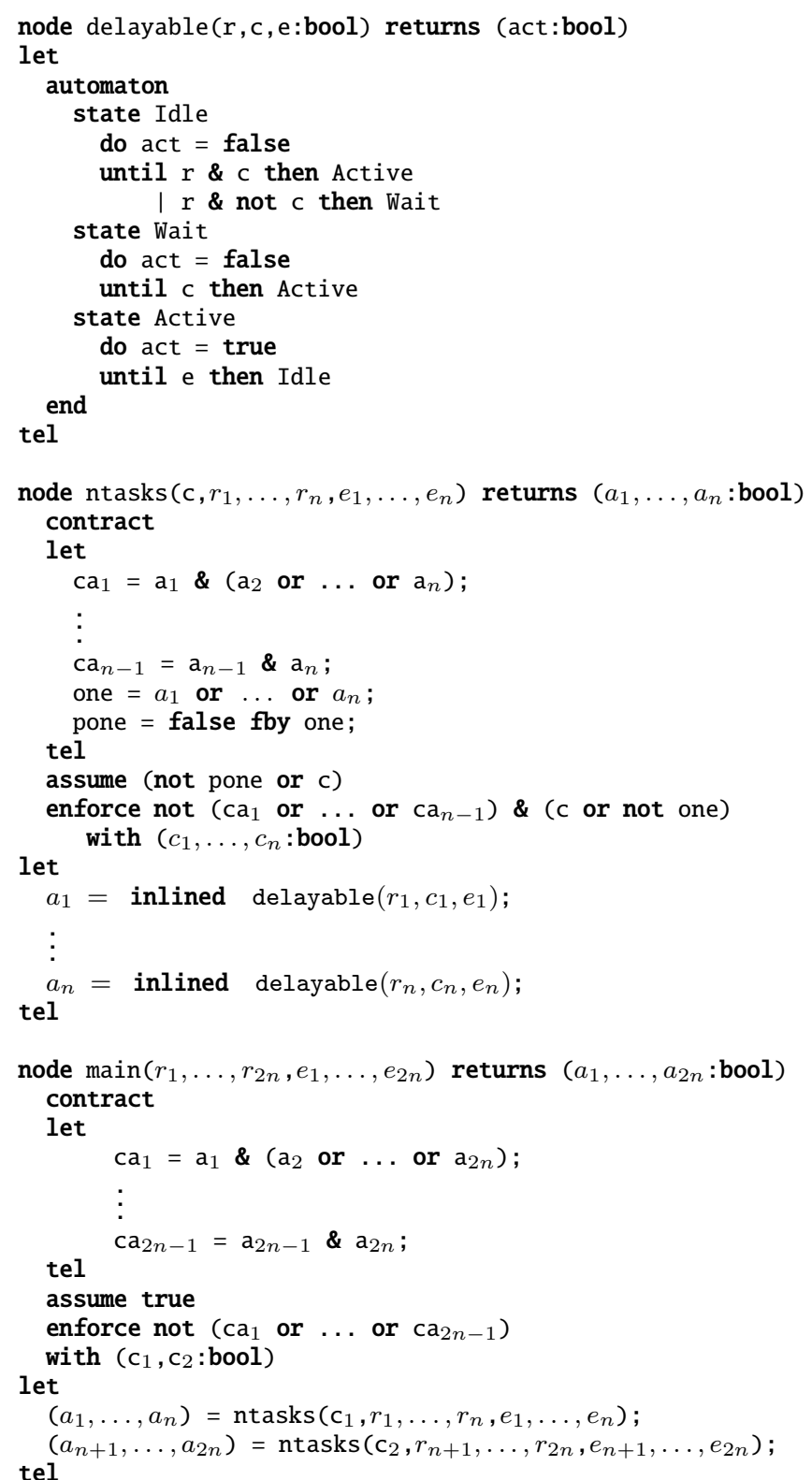

